\title{
Positive Solutions of Two-Point Boundary Value Problems for Monge-Ampère Equations
}

\author{
Baoqiang Yan and Meng Zhang \\ School of Mathematical Sciences, Shandong Normal University, Jinan 250014, China \\ Correspondence should be addressed to Baoqiang Yan; yanbqcn@aliyun.com
}

Received 21 April 2015; Accepted 30 May 2015

Academic Editor: Gennaro Infante

Copyright (C) 2015 B. Yan and M. Zhang. This is an open access article distributed under the Creative Commons Attribution License, which permits unrestricted use, distribution, and reproduction in any medium, provided the original work is properly cited.

\begin{abstract}
This paper considers the following boundary value problem: $\left(\left(-u^{\prime}(t)\right)^{n}\right)^{\prime}=n t^{n-1} f(u(t)), 0<t<1, u^{\prime}(0)=0, u(1)=0$, where $n>1$ is odd. We establish the method of lower and upper solutions for some boundary value problems which generalizes the above equations and using this method we present a necessary and sufficient condition for the existence of positive solutions to the above boundary value problem and some sufficient conditions for the existence of positive solutions.
\end{abstract}

\section{Introduction}

In [1], Kutev obtained the existence of a unique nontrivial solution of the following boundary value problem (BVP)

$$
\begin{aligned}
\left(\left(-u^{\prime}(t)\right)^{n}\right)^{\prime} & =n t^{n-1} f(u(t)), \quad 0<t<1, \\
u^{\prime}(0) & =0, \\
u(1) & =0,
\end{aligned}
$$

where $n>1$ and $f(u)=u^{p}, \forall 0<p \neq n$, which is related to the following Dirichlet problem of the Monge-Ampère equations in $R^{n}$

$$
\begin{aligned}
\operatorname{det}\left(D^{2} u\right) & =\lambda f(-u) \quad \text { in } B, \\
u & =0 \quad \text { on } \partial B,
\end{aligned}
$$

where $B=\left\{x \in R^{n}:|x|<1\right\}$ is the unit ball in $R^{n}$ and $D^{2} u=\left(\partial^{2} u / \partial x_{i} \partial x_{j}\right)$ is the Hessian of $u$.

After Kutev's works, the Monge-Ampère equation has attracted a growing attention in recent years because of its important role in several areas of applied mathematics. For instance, in [2], Hu and Wang obtained some sufficient conditions for the existence and multiplicity of the positive solutions for problem (1) and Dai [3] and Wang [4] discussed the unilateral global bifurcation results for the problem with $f(u)=u^{n}+g(u)$. And there are some results on the existence and multiplicity and nonexistence of nontrivial radial convex solutions of systems of Monge-Ampère equations (see [5-8]) also. We notice that the nonlinearity $f(u)$ is continuous at $u=0$ in the above works. For the case that $f(u)$ is singular at $u=0$, there are fewer results for BVP (1). But some interesting results are presented for BVP (2) in [9-12] where $f(u)$ is singular at $u=0$. We also refer to $[7,8,13,14]$ and references therein for further discussions regarding solutions of the Monge-Ampère equations.

One goal in this paper is to consider the existence of positive solutions under the conditions that $n>1$ and $f(u)$ is singular at $u=0$. Compared to the results in [10], we do not need the monotonicity of $f$.

Our paper is organized as follows. Section 2 lists some lemmas. In Section 3, we obtain a theorem on the upper and lower solutions which is an extension of this method to the class of problem we consider. Section 4 is devoted to our main results on some sufficient conditions for positive solutions of the BVP (1).

\section{Preliminaries}

Let $X$ be the Banach space $C[0,1]$ with $\|v\|=\max _{t \in[0,1]}|v(t)|$. Define a set $K \subseteq X$ by

$$
\begin{aligned}
K & =\{v \in X: v(t) \text { is nonincreasing in } t, v(1) \\
& =0, v(t) \geq\|v\|(1-t) \quad \forall t \in[0,1]\} .
\end{aligned}
$$


It can be easily verified that $K$ is indeed a normal cone in $X$ (see [2]).

Lemma 1 (see $[15,16]$ ). Let $E$ be a Banach space, and let $D \subseteq E$ be a bounded, closed, and convex set. Assume that $F: D \rightarrow D$ is a continuous compact operator. Then, $F$ has at least one fixed point in $D$.

Remark 2. Obviously, assume that $E$ is a Banach space, $D \subseteq E$ is a bounded, closed, and convex set and let $F: E \rightarrow D$ be a continuous compact operator. Then, $F$ has at least one fixed point in $D$ also.

Lemma 3. Assume that function $v \in C[0,1]$ with $v(t) \geq 0$, $v(0)=\|v\|=\max _{t \in[0,1]}|v(t)|$, and $v^{\prime}(t)$ decreasing in $[0,1]$. Then,

$$
v(t) \geq(1-t)\|v\|, \quad t \in[0,1] .
$$

Proof. Since $v^{\prime}(t)$ is decreasing in $[0,1]$, we have

$$
\begin{aligned}
& v(t)-v(0)=\int_{0}^{t} v^{\prime}(s) d s \geq t v^{\prime}(t), \quad \forall t \in[0,1], \\
& v(1)-v(t)=\int_{t}^{1} v^{\prime}(s) d s \leq(1-t) v^{\prime}(t),
\end{aligned}
$$

which implies

$$
\begin{aligned}
&(1-t)(v(t)-v(0))=(1-t) \int_{0}^{t} v^{\prime}(s) d s \\
& \\
& \geq(1-t) t v^{\prime}(t), \quad \forall t \in[0,1], \\
& t(v(1)-v(t))=t \int_{t}^{1} v^{\prime}(s) d s \leq t(1-t) v^{\prime}(t), \\
& \forall t \in[0,1] .
\end{aligned}
$$

Then, for $t \in[0,1]$, we have

$$
(1-t)(v(t)-v(0)) \geq t(v(1)-v(t)) .
$$

That is,

$$
v(t) \geq(1-t) v(0)+t v(1) .
$$

Since $v(0)=\max _{t \in[0,1]}|v(t)|$ and $v(1) \geq 0$, we have

$$
v(t) \geq(1-t)\|v\| \text {. }
$$

The proof is complete.

Remark 4. The idea of the proof of Lemma 3 comes from Lemma 2.2 in [2].

Now, we list some conditions for convenience:

$$
\begin{aligned}
& \left(C_{1}\right) \\
& \quad f:(0, \infty) \longrightarrow(-\infty, \infty) \text { is continuous; } \\
& \left(C_{2}\right) n \text { is odd. }
\end{aligned}
$$

Throughout this paper, we always assume that condition $\left(C_{2}\right)$ holds; that is to say, $n$ is odd.

\section{Upper and Lower Solutions}

Consider the following two-boundary value problem:

$$
\begin{aligned}
\left(\left(-v^{\prime}(t)\right)^{n}\right)^{\prime} & =n t^{n-1} f(v(t)), \quad 0<t<1, \\
v^{\prime}(0) & =a, \\
v(1) & =b,
\end{aligned}
$$

where $f: D \rightarrow R$ is continuous and $D \subseteq R$.

Now, we give following definitions.

Definition 5. If $\alpha \in C[0,1] \cap C^{1}[0,1) \cap C^{2}(0,1)$ and

$$
\begin{aligned}
\left(\left(-\alpha^{\prime}(t)\right)^{n}\right)^{\prime} & \leq n t^{n-1} f(\alpha(t)), \quad 0<t<1, \\
\alpha^{\prime}(0) & =a, \\
\alpha(1) & \leq b,
\end{aligned}
$$

then $\alpha(t)$ is called a lower solution of the BVP (11).

Similarly, we can define an upper solution of BVP (11).

Definition 6. If $\beta \in C[0,1] \cap C^{1}[0,1) \cap C^{2}(0,1)$ and

$$
\begin{aligned}
\left(\left(-\beta^{\prime}(t)\right)^{n}\right)^{\prime} & \geq n t^{n-1} f(\beta(t)), \quad 0<t<1, \\
\beta^{\prime}(0) & =a, \\
\beta(1) & \geq b,
\end{aligned}
$$

then $\beta(t)$ is called an upper solution of BVP (11).

$$
\begin{aligned}
& \text { If } \alpha(t) \leq \beta(t), \text { define } \\
& \qquad D_{\alpha}^{\beta}(t):=\{x \in \mathbb{R}: \alpha(t) \leq x \leq \beta(t) \forall t \in[0,1]\} .
\end{aligned}
$$

Theorem 7. Let $\alpha, \beta \in C[0,1] \cap C^{1}[0,1) \cap C^{2}(0,1)$, respectively, be lower and upper solutions of BVP (11) with $\alpha(t) \leq \beta(t)$ on $[0,1]$ and $D_{\alpha}^{\beta}(t) \subseteq D$ for all $t \in(0,1)$ and

$$
\text { there exists } h \in L^{1}(0,1)
$$

$$
\text { with } \int_{0}^{1} n t^{n-1} h(t) d t<\infty \text {, }
$$

such that $\sup |f(x)| \leq h(t) \quad$ for a.e. $t \in(0,1)$ $x \in D_{\alpha}^{\beta}(t)$

holds. Then, BVP (11) has at least one solution $v \in C[0,1] \cap$ $C^{1}[0,1) \cap C^{2}(0,1)$ and

$$
\alpha(t) \leq v(t) \leq \beta(t), \quad t \in[0,1] .
$$

Proof. Consider the modified boundary value problem of the form

$$
\begin{aligned}
\left(\left(-v^{\prime}(t)\right)^{n}\right)^{\prime} & =n t^{n-1} F(v(t)), \quad 0<t<1, \\
v^{\prime}(0) & =a, \\
v(1) & =b,
\end{aligned}
$$


where the function

$$
\begin{aligned}
& F(v(t)) \\
& = \begin{cases}f(\alpha(t))+\min \{1, \alpha(t)-v(t)\}, & v(t)<\alpha(t), \\
f(v(t)), & \alpha(t) \leq v(t) \leq \beta(t), \\
f(\beta(t))+\min \{1, v(t)-\beta(t)\}, & v(t)>\beta(t) .\end{cases}
\end{aligned}
$$

Obviously, if the problem (17) has a solution $v(t)$ with $\alpha(t) \leq v(t) \leq \beta(t), t \in[0,1]$, then problem (11) has a solution $v(t), t \in[0,1]$.

For $v \in X=C[0,1]$, define an operator

$$
\begin{aligned}
(A v)(t)= & b+(t-1) a \\
& +\int_{t}^{1}\left(\int_{0}^{s} n \tau^{n-1} F(v(\tau)) d \tau\right)^{1 / n} d s, \\
& 0 \leq t \leq 1 .
\end{aligned}
$$

A standard argument shows that $A: X \rightarrow\{v:\|v\| \leq|a|+$ $\left.|b|+\left(\int_{0}^{1} n \tau^{n-1}[h(\tau)+1] d \tau\right)^{1 / n}\right\}$ is continuous and compact. Lemma 1 guarantees that $A$ has a fixed point $v \in C[0,1] \cap$ $C^{1}[0,1) \cap C^{2}(0,1)$; that is BVP $(17)$ has a solution $v(t)$.

Now, we claim that

$$
\alpha(t) \leq v(t) \leq \beta(t), \quad t \in[0,1],
$$

which guarantees that $v \in C[0,1] \cap C^{1}[0,1) \cap C^{2}(0,1)$ is a solution of BVP (11).

Let $x(t)=\alpha(t)-v(t), t \in[0,1]$.

First, we show that

$$
x(0) \leq 0, \quad \text { that is, } \alpha(0) \leq v(0) .
$$

Suppose that $x(0)>0$. Then, $\exists t_{0}>0$ such that $x(t)>0$, $t \in\left[0, t_{0}\right]$. Let $t_{*}=\sup \{t: x(s)>0, s \in[0, t)\}$. Obviously, $t_{*} \leq 1$ and

$$
\alpha(t)>v(t), \quad t \in\left[0, t_{*}\right), x\left(t_{*}\right)=0 .
$$

Let $y(t)=\left(-\alpha^{\prime}(t)\right)^{n}-\left(-v^{\prime}(t)\right)^{n}, t \in\left(0, t_{*}\right)$. Then

$$
\begin{aligned}
y^{\prime}(t)= & \left(\left(-\alpha^{\prime}(t)\right)^{n}\right)^{\prime}-\left(\left(-v^{\prime}(t)\right)^{n}\right)^{\prime} \\
\leq & n t^{n-1} f(\alpha(t)) \\
& -\left(n t^{n-1} f(\alpha(t))+\min \{1, \alpha(t)-v(t)\}\right) \\
< & 0, \quad t \in\left(0, t_{*}\right),
\end{aligned}
$$

which implies that $y(t)$ is decreasing on $\left[0, t_{*}\right)$. And so $y(t)<$ $y(0)$ for all $t \in\left(0, t_{*}\right)$; that is,

$$
\left(-\alpha^{\prime}(t)\right)^{n}-\left(-v^{\prime}(t)\right)^{n}<\left(-\alpha^{\prime}(0)\right)^{n}-\left(-v^{\prime}(0)\right)^{n}=0 .
$$

That is,

$$
\left(-\alpha^{\prime}(t)\right)^{n}<\left(-v^{\prime}(t)\right)^{n}, \quad t \in\left(0, t_{*}\right) .
$$

From $\left(C_{2}\right)$, we have

$$
-\alpha^{\prime}(t)<-v^{\prime}(t) .
$$

That is,

$$
x^{\prime}(t)=\alpha^{\prime}(t)-v^{\prime}(t)>0,
$$

which means that $x(t)$ is increasing on $\left[0, t_{*}\right)$. Then, $x(t)>$ $x(0)>0, t \in\left(0, t_{*}\right)$. The continuity of $x(t)$ at $t=t_{*}$ means that $x\left(t_{*}\right) \geq x(0)>0$, which contradicts (22).

Secondly, we prove that

$$
\alpha(t) \leq v(t), \quad \forall t \in[0,1] .
$$

Assume that $v(t)<\alpha(t)$ for some $t \in(0,1)$. Since $\alpha(0) \leq$ $v(0)$ and $\alpha(1) \leq v(1)$, there exists a $\tilde{t} \in(0,1)$ such that $x(\widetilde{t})=$ $\max _{t \in[0,1]} x(t)=\max _{t \in[0,1]}(\alpha(t)-v(t))>0$ with $x^{\prime}(\widetilde{t})=0$ $\left(\right.$ note $\left.\alpha^{\prime}(\widetilde{t})=v^{\prime}(\widetilde{t})\right)$. Then,

$$
\begin{aligned}
\left(\left(-v^{\prime}(\widetilde{t})\right)^{n}\right)^{\prime}= & n \widetilde{t}^{n-1} F(v(\widetilde{t})) \\
= & n \widetilde{t}^{n-1} f(\alpha(\widetilde{t})) \\
& +n \widetilde{t}^{n-1} \min \{1, \alpha(\widetilde{t})-v(\widetilde{t})\} \\
> & n \widetilde{t}^{n-1} f(\alpha(\widetilde{t})) \geq\left(\left(-\alpha^{\prime}(\widetilde{t})\right)^{n}\right)^{\prime} .
\end{aligned}
$$

From the continuity of $\left(\left(-v^{\prime}(t)\right)^{n}\right)^{\prime}$ and $\left(\left(-\alpha^{\prime}(t)\right)^{n}\right)^{\prime}$ in $(0,1)$, there exists a $\delta>0$ such that

$$
\begin{aligned}
\left(\left(-v^{\prime}(t)\right)^{n}\right)^{\prime}>\left(\left(-\alpha^{\prime}(t)\right)^{n}\right)^{\prime}, & \\
t & \in(\widetilde{t}-\delta, \widetilde{t}+\delta) \subseteq(0,1) .
\end{aligned}
$$

Integrating both sides of the above inequality from $\tilde{t}$ to $t \in$ $(\widetilde{t}, \widetilde{t}+\delta)$, we get

$$
\begin{array}{r}
\left(-v^{\prime}(t)\right)^{n}-\left(-v^{\prime}(\widetilde{t})\right)^{n}>\left(-\alpha^{\prime}(t)\right)^{n}-\left(-\alpha^{\prime}(\widetilde{t})\right)^{n}, \\
t \in(\widetilde{t}, \widetilde{t}+\delta) .
\end{array}
$$

That is,

$$
\left(-v^{\prime}(t)\right)^{n}>\left(-\alpha^{\prime}(t)\right)^{n}, \quad t \in(\widetilde{t}, \tilde{t}+\delta) .
$$

From $\left(C_{2}\right)$, we have

$$
-v^{\prime}(t)>-\alpha^{\prime}(t), \quad t \in(\tilde{t}, \tilde{t}+\delta) .
$$

And so

$$
\alpha^{\prime}(t)-v^{\prime}(t)>0, \quad t \in(\widetilde{t}, \tilde{t}+\delta) .
$$

Then,

$$
x^{\prime}(t)>0, \quad t \in(\widetilde{t}, \tilde{t}+\delta),
$$

which contradicts the fact that $x(\widetilde{t})=\max _{t \in[0,1]} x(t)$. Hence, (28) is true.

The same argument shows that

$$
v(t) \leq \beta(t), \quad \forall t \in[0,1] .
$$

Consequently, (28) and (36) show that (20) holds. Then, $v(t)$ is a solution to BVP (11).

Remark 8. The idea of the proof comes from $[17,18]$. 


\section{Main Results}

Suppose that $K$ is defined in Section 2. Now, we list the following condition for convenience:

$\left(H_{1}\right) f:(0,+\infty) \rightarrow(0,+\infty)$, continuous and $\exists \lambda, \mu, \delta,(-\infty<\lambda<0<\mu<1,0<\delta \leq 1)$, $\forall v \in(0,+\infty)$ such that

$$
\begin{array}{ll}
c_{0}^{\mu} f(v) \leq f\left(c_{0} v\right) \leq c_{0}^{\lambda} f(v), & 0 \leq c_{0} \leq \delta, \\
c_{0}^{\lambda} f(v) \leq f\left(c_{0} v\right) \leq c_{0}^{\mu} f(v), & c_{0} \geq \frac{1}{\delta} .
\end{array}
$$

Remark 9. Assume that

$$
f(v)=v^{-\alpha_{1}}+v^{-\alpha_{2}}+v^{\beta_{1}}+v^{\beta_{2}}, \quad v \in(0,+\infty),
$$

where $\alpha_{1}, \alpha_{2} \in(0,+\infty), \beta_{1}, \beta_{2} \in(0,1)$ are constants. Let $\delta=$ $1, \lambda=\min \left\{-\alpha_{1},-\alpha_{2}\right\}, \mu=\max \left\{\beta_{1}, \beta_{2}\right\}$. Then, if $0 \leq c_{0} \leq \delta$, we have

$$
\begin{aligned}
f\left(c_{0} v\right)= & \left(c_{0} v\right)^{-\alpha_{1}}+\left(c_{0} v\right)^{-\alpha_{2}}+\left(c_{0} v\right)^{\beta_{1}}+\left(c_{0} v\right)^{\beta_{2}} \\
= & \left(c_{0}\right)^{-\alpha_{1}} v^{-\alpha_{1}}+\left(c_{0}\right)^{-\alpha_{2}} v^{-\alpha_{2}}+\left(c_{0}\right)^{\beta_{1}} v^{\beta_{1}} \\
& +\left(c_{0}\right)^{\beta_{2}} v^{\beta_{2}} \\
\leq & \left(c_{0}\right)^{\lambda}\left(v^{-\alpha_{1}}+v^{-\alpha_{2}}+v^{\beta_{1}}+v^{\beta_{2}}\right) \\
= & c^{\lambda} f(v), \\
f\left(c_{0} v\right)= & \left(c_{0} v\right)^{-\alpha_{1}}+\left(c_{0} v\right)^{-\alpha_{2}}+\left(c_{0} v\right)^{\beta_{1}}+\left(c_{0} v\right)^{\beta_{2}} \\
= & \left(c_{0}\right)^{-\alpha_{1}} v^{-\alpha_{1}}+\left(c_{0}\right)^{-\alpha_{2}} v^{-\alpha_{2}}+\left(c_{0}\right)^{\beta_{1}} v^{\beta_{1}} \\
& +\left(c_{0}\right)^{\beta_{2}} v^{\beta_{2}} \\
\geq & \left(c_{0}\right)^{\mu}\left(v^{-\alpha_{1}}+v^{-\alpha_{2}}+v^{\beta_{1}}+v^{\beta_{2}}\right) \\
= & c^{\mu} f(v) .
\end{aligned}
$$

A similar argument shows that if $c_{0} \geq 1 / \delta$, we have

$$
c_{0}^{\lambda} f(v) \leq f\left(c_{0} v\right) \leq c_{0}^{\mu} f(v) .
$$

We have the following theorems.

Theorem 10. Suppose that $\left(H_{1}\right)$ holds and $2(\mu-\lambda) /(1-\mu)<1$. Then, the necessary and sufficient condition of the existence of the positive solution in $C^{1}[0,1] \cap C^{2}(0,1)$ for $B V P(1)$ is

$$
0<\int_{0}^{1} t^{n-1} f(1-t) d t<\infty
$$

Proof.

Necessity. Suppose that $v \in C^{1}[0,1] \cap C^{2}(0,1)$ is a positive solution. We have

$$
\begin{aligned}
& n\left(-v^{\prime}(t)\right)^{n-1}\left(-v^{\prime \prime}(t)\right)=n t^{n-1} f(v(t))>0, \\
& t \in(0,1), \\
& -\infty<v^{\prime}(t)=-\left(\int_{0}^{t} n \tau^{n-1} f(v(\tau)) d \tau\right)^{1 / n}<0, \\
& \left.v(t)=\int_{t}^{1}\left(\int_{0}^{s} n \tau^{n-1} f(v(\tau)) d \tau\right)^{1 / n} d s, 1\right], \\
& \quad \leq(1-t)\left(\int_{0}^{1} n \tau^{n-1} f(v(\tau)) d \tau\right)^{1 / n} .
\end{aligned}
$$

Let

$$
M_{v}=\left(\int_{0}^{1} n \tau^{n-1} f(v(\tau)) d \tau\right)^{1 / n}
$$

From $v(t)>0$ and $v(1)=0$, Lemma 3 implies that $v \in K$ and

$$
\|v\|(1-t) \leq v(t) \leq M_{v}(1-t) .
$$

Let $c_{1}>0$ be a constant such that $c_{1} M_{v} \leq \delta, c_{1} \leq \delta$. By (37) and (44), we have

$$
\begin{aligned}
f(v(t)) & =f\left(\frac{1}{c_{1}} \frac{c_{1} v(t)}{1-t}(1-t)\right) \\
& \geq\left(\frac{1}{c_{1}}\right)^{\lambda} f\left(\frac{c_{1} v(t)}{1-t}(1-t)\right) \\
& \geq c_{1}^{\mu-\lambda}\left(\frac{v(t)}{1-t}\right)^{\mu} f(1-t) \\
& \geq c_{1}^{\mu-\lambda}\|v\|^{\mu} f(1-t), \quad t \in(0,1) .
\end{aligned}
$$

That is,

$$
n t^{n-1} f(v(t)) \geq n c_{1}^{\mu-\lambda}\|v\|^{\mu} t^{n-1} f(1-t), \quad t \in(0,1) .
$$

Since

$$
\left(-v^{\prime}(1)\right)^{n}=\int_{0}^{1} n s^{n-1} f(v(s)) d s<+\infty
$$

we have from (46)

$$
\begin{aligned}
0 & <\int_{0}^{1} n t^{n-1} f(1-t) d t \\
& \leq \int_{0}^{1} n c_{1}^{\lambda-\mu}\|v\|^{-\mu} t^{n-1} f(v(t)) d t \\
& =c_{1}^{\lambda-\mu}\|v\|^{-\mu}\left(-v^{\prime}(1)\right)^{n}<+\infty .
\end{aligned}
$$

Hence, (41) holds. 
Sufficiency. Since (41) holds, set

$$
h(t):=\int_{t}^{1}\left(\int_{0}^{s} n \tau^{n-1} f(1-\tau) d \tau\right)^{1 / n} d s, \quad 0 \leq t \leq 1 .
$$

Obviously $h(t) \geq 0$ and $h^{\prime}(t)$ are decreasing in $[0,1]$. Then, Lemma 3 means that $h(t) \geq\|h\|(1-t)$. From (41), we know that there exists $N>0$ such that $\int_{0}^{1} n t^{n-1} f(1-t) d t \leq N$. Consequently,

$$
a_{1}(1-t) \leq h(t) \leq a_{2}(1-t)
$$

where $a_{1}=\min \{1,\|h\|\}, a_{2}=\max \left\{1, N^{1 / n}\right\}$.

Let $\alpha(t):=k_{1} h(t)$ and $\beta(t):=k_{2} h(t)$, where $c_{2} \geq 1 / a_{1} \delta$ is big enough such that $k_{1}=(1 / 2)\left(c_{2}^{\lambda-\mu} a_{1}^{\lambda}\right)^{1 /(1-\mu)} \leq 1 / 2, k_{2}=$ $2\left(c_{2}^{\mu-\lambda} a_{1}^{\mu}\right)^{1 /(1-\mu)} \geq 2$. Obviously,

$$
0 \leq \alpha(t) \leq \beta(t), \quad t \in[0,1] .
$$

Since (note (50))

$$
\begin{aligned}
f\left(c_{2} h(t)\right) & =f\left(\frac{c_{2} h(t)}{1-t}(1-t)\right) \\
\geq c_{2}^{\lambda}\left(\frac{h(t)}{1-t}\right)^{\lambda} f(1-t) \geq c_{2}^{\lambda} a_{1}^{\lambda} f(1-t), & t \in(0,1),
\end{aligned}
$$

we have (note $k_{1} / c_{2} \leq \delta$ )

$$
\begin{aligned}
f(\alpha(t)) & =f\left(k_{1} h(t)\right) \geq\left(\frac{k_{1}}{c_{2}}\right)^{\mu} f\left(c_{2} h(t)\right) \\
& \geq k_{1}^{\mu} c_{2}^{\lambda-\mu} a_{1}^{\lambda} f(1-t) .
\end{aligned}
$$

From

$$
k_{1}=\frac{1}{2}\left(c_{2}^{\lambda-\mu} a_{1}^{\lambda}\right)^{1 /(1-\mu)}
$$

we can obtain

$$
k_{1}^{1-\mu}=\left(\frac{1}{2}\right)^{1-\mu} c_{2}^{\lambda-\mu} a_{1}^{\lambda}
$$

That is,

$$
c_{2}^{\lambda-\mu} a_{1}^{\lambda}=k_{1}^{1-\mu}\left(\frac{1}{2}\right)^{\mu-1} .
$$

And so (53) is

$$
\begin{aligned}
f(\alpha(t)) & \geq\left(\frac{1}{2}\right)^{\mu-1} k_{1}^{1-\mu} k_{1}^{\mu} f(1-t) \geq k_{1}^{1-\mu} k_{1}^{\mu} f(1-t) \\
& =k_{1} f(1-t), \quad t \in(0,1) .
\end{aligned}
$$

Similarly, we have

$$
\begin{aligned}
f(\beta(t)) & =f\left(k_{2} h(t)\right) \leq\left(\frac{1}{c_{2}}\right)^{\lambda} f\left(c_{2} k_{2} h(t)\right) \\
& \leq k_{2} f(1-t) .
\end{aligned}
$$

Differentiating $\alpha(t)$ on $(0,1)$, we have

$$
\begin{aligned}
\alpha^{\prime}(t) & =-k_{1}\left(\int_{0}^{t} n \tau^{n-1} f(1-\tau) d \tau\right)^{1 / n}, \\
\left(-\alpha^{\prime}(t)\right)^{n} & =k_{1}^{n} \int_{0}^{t} n \tau^{n-1} f(1-\tau) d \tau, \\
\left(\left(-\alpha^{\prime}(t)\right)^{n}\right)^{\prime} & =k_{1}^{n} n t^{n-1} f(1-t) \leq k_{1} n t^{n-1} f(1-t) \\
& \leq n t^{n-1} f(\alpha(t)),
\end{aligned}
$$

with $\alpha^{\prime}(0)=0, \alpha(1)=0$, which implies that $\alpha(t)$ is a lower solution to BVP (1).

Similarly, we have

$$
\left(\left(-\beta^{\prime}(t)\right)^{n}\right)^{\prime} \geq n t^{n-1} f(\beta(t)),
$$

with $\beta^{\prime}(0)=0, \beta(1)=0$. Hence, $\beta(t)$ is an upper solution to BVP (1).

Theorem 7 guarantees that BVP (1) has at least one positive solution in $C^{1}[0,1] \cap C^{2}(0,1)$.

Remark 11. Idea of the proof comes from [19].

Theorem 12. Assume that $\left(C_{1}\right)$ holds and the following conditions are true:

(1) there exist $L>0$ and $\varepsilon>0$ such that

$$
f(x)>L, \quad \forall x \in(0, \varepsilon]
$$

(2) there exists $M>0$ such that

$$
\sup _{x \geq M}|f(x)|<+\infty
$$

Then, BVP (1) has at least one positive solution $v \in C[0,1] \cap$ $C^{1}[0,1) \cap C^{2}(0,1)$. If, moreover, $f$ is decreasing, then the solution is unique.

Proof. By assumption (1), there is $\varepsilon_{m}>0$ such that

$$
f(x)>L, \quad \forall x \in\left(0, \varepsilon_{m}\right] .
$$

Without restriction, we can assume that $\left\{\varepsilon_{m}\right\}$ is a decreasing sequence and $\lim _{m \rightarrow+\infty} \varepsilon_{m}=0$ with $\varepsilon_{m}<\min \{1, L\}$ for all $m \in \mathbb{N}$.

Let

$$
\gamma(t):=\int_{t}^{1}\left(\int_{0}^{s} n \tau^{n-1}(1-\tau) d \tau\right)^{1 / n} d s, \quad t \in[0,1] .
$$

It is easy to see that

$$
0<\gamma(t), \quad t \in[0,1), \gamma \in C^{2}[0,1]
$$

Let

$$
k_{0}:=\frac{1}{2+\|\gamma\|} \varepsilon_{1}
$$


which guarantees that

$$
0 \leq k_{0} \gamma(t)<\varepsilon_{1}, \quad t \in[0,1] .
$$

Set

$$
\alpha_{1}(t): \equiv \varepsilon_{1}, \quad t \in[0,1] .
$$

From (63), we have

$$
\begin{aligned}
\left(\left(-\alpha_{1}^{\prime}(t)\right)^{n}\right)^{\prime} & =0<n t^{n-1} L<n t^{n-1} f\left(\varepsilon_{1}\right) \\
& =n t^{n-1} f\left(\alpha_{1}(t)\right), \quad t \in(0,1), \\
\alpha_{1}^{\prime}(0) & =0, \\
\alpha_{1}(1) & =\varepsilon_{1} .
\end{aligned}
$$

From $\left(C_{1}\right)$ and condition (2), we choose an $M^{\prime}>\max \left\{\varepsilon_{1}, M\right\}$ such that

$$
\sup _{x \in\left[M^{\prime},+\infty\right)}|f(x)|=R<+\infty .
$$

Let

$$
\beta_{1}(t):=M^{\prime}+\int_{t}^{1}\left(\int_{0}^{s} n \tau^{n-1}\left(M^{\prime}+R+1\right) d \tau\right)^{1 / n} d s
$$

$$
t \in[0,1] .
$$

Equation (70) guarantees that

$$
\begin{aligned}
f\left(\beta_{1}(t)\right) & \leq R, \quad \forall t \in[0,1], \\
\left(\left(-\beta_{1}^{\prime}(t)\right)^{n}\right)^{\prime} & =n t^{n-1}\left(M^{\prime}+R+1\right) \\
& \geq n t^{n-1} f\left(\beta_{1}(t)\right), \quad t \in(0,1), \\
\beta_{1}^{\prime}(0) & =0, \\
\beta_{1}(1) & =M^{\prime} \geq \varepsilon_{1} .
\end{aligned}
$$

Now, Theorem 7 implies that there exists $u_{1} \in C[0,1] \cap$ $C^{1}[0,1) \cap C^{2}(0,1)$ such that

$$
\begin{aligned}
\left(\left(-u_{1}^{\prime}(t)\right)^{n}\right)^{\prime} & =n t^{n-1} f\left(u_{1}(t)\right), \quad t \in(0,1), \\
u_{1}^{\prime}(0) & =0, \\
u_{1}(1) & =\varepsilon_{1} .
\end{aligned}
$$

Let $\alpha_{2}(t):=\varepsilon_{2}, t \in[0,1]$, and $\beta_{2}(t):=u_{1}(t), t \in[0,1]$. It is easy to prove that

$$
\begin{aligned}
\left(\left(-\alpha_{2}^{\prime}(t)\right)^{n}\right)^{\prime} & =0<n t^{n-1} L<n t^{n-1} f\left(\varepsilon_{2}\right) \\
& =n t^{n-1} f\left(\alpha_{2}(t)\right), \quad t \in(0,1), \\
\alpha_{2}^{\prime}(0) & =0, \\
\alpha_{2}(1) & =\varepsilon_{2}, \\
\left(\left(-\beta_{2}^{\prime}(t)\right)^{n}\right)^{\prime} & =n t^{n-1} f\left(\beta_{2}(t)\right), \quad t \in(0,1), \\
\beta_{2}^{\prime}(0) & =0, \\
\beta_{2}(1) & =\varepsilon_{1}>\varepsilon_{2} .
\end{aligned}
$$

Theorem 7 guarantees that there exists $u_{2} \in C[0,1] \cap C^{1}[0,1)$ $n C^{2}(0,1)$ such that

$$
\begin{aligned}
\left(\left(-u_{2}^{\prime}(t)\right)^{n}\right)^{\prime} & =n t^{n-1} f\left(u_{2}(t)\right), \quad t \in(0,1), \\
u_{2}^{\prime}(0) & =0 \\
u_{2}(1) & =\varepsilon_{2} .
\end{aligned}
$$

Proceeding the above proof, we can get a sequence $\left\{u_{m}(\cdot)\right\}$ such that

$$
\begin{aligned}
\varepsilon_{m} & \leq u_{m}(t) \leq u_{m-1}(t), \quad t \in[0,1], \\
\left(\left(-u_{m}^{\prime}(t)\right)^{n}\right)^{\prime} & =n t^{n-1} f\left(u_{m}(t)\right), \quad t \in(0,1), \\
u_{m}^{\prime}(0) & =0, \\
u_{m}(1) & =\varepsilon_{m} .
\end{aligned}
$$

Now, we claim that

$$
u_{m}(t) \geq k_{0} \gamma(t), \quad t \in[0,1], m \in \mathbb{N} .
$$

First, we show that

$$
\begin{aligned}
& u_{m}(0) \geq k_{0} \gamma(0), \\
& u_{m}(1) \geq k_{0} \gamma(1),
\end{aligned}
$$

$m \in \mathbb{N}$.

Obviously, $u_{m}(1)=\varepsilon_{m}>0=k_{0} \gamma(1)$. Suppose $u_{m}(0)<$ $k_{0} \gamma(0)$. Let $h(t):=u_{m}(t)-k_{0} \gamma(t), t \in[0,1]$, and $t^{\prime}=\sup \{t$ : $h(s)<0, s \in[0, t)\}$. Obviously, $t^{\prime}<1$ (note $u_{m}(1) \geq k_{0} \gamma(1)$ ) and $h\left(t^{\prime}\right)=0, h(t)<0$, for all $t \in\left[0, t^{\prime}\right)$, which implies that

$$
\varepsilon_{m} \leq u_{m}(t)<k_{0} \gamma(t)<\varepsilon_{1}, \quad t \in\left[0, t^{\prime}\right] .
$$

For $t \in\left(0, t^{\prime}\right)$, condition (1) guarantees that

$$
\begin{aligned}
\left(\left(-u_{m}^{\prime}(t)\right)^{n}\right)^{\prime} & =n t^{n-1} f\left(u_{m}(t)\right)>n t^{n-1} L \\
& >n t^{n-1} k_{0}(1-t)=\left(\left(-k_{0} \gamma^{\prime}(t)\right)^{n}\right)^{\prime} .
\end{aligned}
$$

Integrate (80) from 0 to $t \in\left(0, t^{\prime}\right)$ to obtain

$$
\left(-u_{m}^{\prime}(t)\right)^{n}>\left(-k_{0} \gamma^{\prime}(t)\right)^{n}, \quad t \in\left(0, t^{\prime}\right) .
$$

From $\left(C_{2}\right)$, we have

$$
-u_{m}^{\prime}(t)>-k_{0} \gamma^{\prime}(t), \quad t \in\left(0, t^{\prime}\right) .
$$

That is,

$$
h^{\prime}(t)=u_{m}^{\prime}(t)-k_{0} \gamma^{\prime}(t)<0, \quad t \in\left(0, t^{\prime}\right),
$$

which means that $h(t)$ is decreasing on $\left(0, t^{\prime}\right]$. The continuity of $h(t)$ at $t^{\prime}$ means that $h\left(t^{\prime}\right)<0$. This contradicts that $h\left(t^{\prime}\right)=$ 0 . Hence, (78) is true. 
Secondly, we show that

$$
u_{m}(t) \geq k_{0} \gamma(t), \quad \forall t \in[0,1], m \in \mathbb{N} .
$$

Suppose that there is a $t \in(0,1)$ such that $h(t)<0$. There exists a $t_{0} \in(0,1)$ with $h\left(t_{0}\right)=\min _{t \in[0,1]} h(t)<0$ and $h^{\prime}\left(t_{0}\right)=0$; that is, $u_{m}^{\prime}\left(t_{0}\right)=k_{0} \gamma^{\prime}\left(t_{0}\right)$. Now, $h\left(t_{0}\right)<0$ implies that $\varepsilon_{m} \leq u_{m}\left(t_{0}\right)<k_{0} \gamma\left(t_{0}\right)<\varepsilon_{1}$, which together with condition (1) guarantees that

$$
\begin{aligned}
\left(\left(-u_{m}^{\prime}\left(t_{0}\right)\right)^{n}\right)^{\prime} & =n t_{0}^{n-1} f\left(u_{m}\left(t_{0}\right)\right)>n t_{0}^{n-1} L \\
& >n t_{0}^{n-1} k_{0}\left(1-t_{0}\right) \\
& =\left(\left(-k_{0} \gamma^{\prime}\left(t_{0}\right)\right)^{n}\right)^{\prime} .
\end{aligned}
$$

The continuity of $\left(\left(-u_{m}^{\prime}(t)\right)^{n}\right)^{\prime}$ and $\left(\left(-k_{0} \gamma^{\prime}(t)\right)^{n}\right)^{\prime}$ at $t=t_{0}$ guarantees that there exists a $\delta>0$ such that

$$
\begin{aligned}
\left(\left(-u_{m}^{\prime}(t)\right)^{n}\right)^{\prime}>\left(\left(-k_{0} \gamma^{\prime}(t)\right)^{n}\right)^{\prime}, & \\
& t \in\left(t_{0}-\delta, t_{0}+\delta\right) .
\end{aligned}
$$

Integrate (86) from $t_{0}$ to $t \in\left(t_{0}, t_{0}+\delta\right)$ to obtain

$$
\left(-u_{m}^{\prime}(t)\right)^{n}>\left(-k_{0} \gamma^{\prime}(t)\right)^{n}, \quad t \in\left(t_{0}, t_{0}+\delta\right) .
$$

From $\left(C_{2}\right)$, we have

$$
-u_{m}^{\prime}(t)>-k_{0} \gamma^{\prime}(t), \quad t \in\left(t_{0}, t_{0}+\delta\right) .
$$

That is,

$$
h^{\prime}(t)=u_{m}^{\prime}(t)-k_{0} \gamma^{\prime}(t)<0, \quad t \in\left(t_{0}, t_{0}+\delta\right),
$$

which means that $h(t)$ is decreasing on $\left(t_{0}, t_{0}+\delta\right)$. This contradicts that $h\left(t_{0}\right)=\min _{t \in[0,1]} h(t)$. Consequently, (84) is true.

Consider now the pointwise limit as follows:

$$
u_{*}(t):=\lim _{m \rightarrow+\infty} u_{m}(t), \quad t \in[0,1]
$$

It is clear that

$$
k_{0} \gamma(t) \leq u_{*}(t) \leq u_{m}(t), \quad \forall t \in[0,1],
$$

which means that

$$
u_{*}(1)=0 \text {. }
$$

For any $1>\epsilon>0$, from (91) and

$$
\left(\left(-u_{m}^{\prime}(t)\right)^{n}\right)^{\prime}=n t^{n-1} f\left(u_{m}(t)\right), \quad \forall t \in(0,1),
$$

we have

$$
\begin{aligned}
& \sup \left\{n t^{n-1} f(x): t \in[0,1-\epsilon], k_{0} \gamma(t) \leq x \leq u_{1}(t)\right\} \\
& <+\infty
\end{aligned}
$$

That is, $\forall t \in[0,1-\epsilon]$

$$
\begin{aligned}
& \left|\left(\left(-u_{m}^{\prime}(t)\right)^{n}\right)^{\prime}\right| \leq \sup \left\{n t^{n-1} f(x): t\right. \\
& \left.\quad \in[0,1-\epsilon], k_{0} \gamma(t) \leq x \leq u_{1}(t)\right\}<+\infty .
\end{aligned}
$$

The Ascoli-Arzela theorem guarantees that $\left\{u_{m}(t)\right\}$ converges uniformly $u_{*}(t)$ on $[0,1-\epsilon]$ and $\left\{u_{m}^{\prime}(t)\right\}$ converges uniformly $u_{*}^{\prime}(t)$ on $[0,1-\epsilon]$. Since $\epsilon>0$ is arbitrary, we find that

$$
\begin{aligned}
u_{*} & \in C^{1}[0,1), \\
\left(\left(-u_{*}^{\prime}(t)\right)^{n}\right)^{\prime} & =n t^{n-1} f\left(u_{*}(t)\right), \quad t \in(0,1), \\
u_{*}^{\prime}(0) & =0 .
\end{aligned}
$$

It remains only to prove the continuity of $u_{*}(t)$ at $t=1$.

Let $\varepsilon>0$ be given. Take $u_{m_{\varepsilon}}(t)$ with $u_{m_{\varepsilon}}(0)<\varepsilon$. Since $u_{m_{\varepsilon}}(t)$ is continuous at $t=1$, we can find $\delta>0$ such that

$$
0<u_{m_{\varepsilon}}(t)<\varepsilon, \quad \forall t \in[1-\delta, 1] .
$$

From (91), we obtain that

$$
0<u_{*}(t)<\varepsilon, \quad t \in[1-\delta, 1],
$$

which together with (92) means that $u_{*}(t)$ is continuous at $t=1$. From (92) and (96), $u_{*}$ is positive solution to BVP (1) in $C^{1}[0,1) \cap C^{2}(0,1)$.

Now, we show that the uniqueness of $u_{*}$ follows when $f$ is decreasing on $(0,+\infty)$.

Suppose that, by contradiction, $v_{1}$ and $v_{2}$ are two different solutions. Obviously

$$
v_{1}(1)=v_{2}(1)=0 .
$$

First, we show that

$$
v_{1}(0)=v_{2}(0) \text {. }
$$

In fact, if $v_{1}(0)<v_{2}(0)$, let $t^{*}=\sup \left\{t: v_{1}(s)<v_{2}(s), s \in\right.$ $(0, t)\}$. Obviously $1 \geq t^{*}>0, v_{1}(t)<v_{2}(t)$ for all $t \in$ $\left[0, t^{*}\right]$ and $v_{1}\left(t^{*}\right)=v_{2}\left(t^{*}\right)$ (notice $v_{1}(1)=v_{2}(1)=0$ ). The monotonicity of $f$ implies that

$$
\begin{aligned}
\left(\left(-v_{1}^{\prime}(t)\right)^{n}\right)^{\prime} & =n t^{n-1} f\left(v_{1}(t)\right)>n t^{n-1} f\left(v_{2}(t)\right) \\
& =\left(\left(-v_{2}^{\prime}(t)\right)^{n}\right)^{\prime}, \quad \forall t \in\left(0, t^{*}\right) .
\end{aligned}
$$

Integrate (101) from 0 to $t$ to obtain

$$
\left(-v_{1}^{\prime}(t)\right)^{n}>\left(-v_{2}^{\prime}(t)\right)^{n}, \quad \forall t \in\left(0, t^{*}\right) .
$$

From $\left(C_{2}\right)$, we have

$$
-v_{1}^{\prime}(t)>-v_{2}^{\prime}(t), \quad \forall t \in\left(0, t^{*}\right) .
$$

That is,

$$
\left(v_{1}(t)-v_{2}(t)\right)^{\prime}<0, \quad \forall t \in\left(0, t^{*}\right) .
$$


Then, $v_{1}(t)-v_{2}(t)$ is decreasing on $\left(0, t^{*}\right)$, which together with $v_{1}(0)-v_{2}(0)<0$ means that $v_{1}\left(t^{*}\right)-v_{2}\left(t^{*}\right)<0$. This is a contradiction. Hence, (100) is true.

Secondly, we show that

$$
v_{1}(t) \equiv v_{2}(t), \quad t \in(0,1)
$$

Suppose that there exists $t_{0} \in(0,1)$ with $v_{1}\left(t_{0}\right) \neq v_{2}\left(t_{0}\right)$. Without loss of generality, we suppose that $v_{1}\left(t_{0}\right)<v_{2}\left(t_{0}\right)$. Let $t^{\prime}=\min _{t \in[0,1]}\left(v_{1}(t)-v_{2}(t)\right)$. Equation (100) guarantees that $t^{\prime} \in(0,1), v_{1}\left(t^{\prime}\right)<v_{2}\left(t^{\prime}\right)$, and $v_{1}^{\prime}\left(t^{\prime}\right)=v_{2}^{\prime}\left(t^{\prime}\right)$. The monotonicity of $f$ implies that

$$
\begin{aligned}
\left(\left(-v_{1}^{\prime}\left(t^{\prime}\right)\right)^{n}\right)^{\prime} & =n t^{\prime n-1} f\left(v_{1}\left(t^{\prime}\right)\right) \\
& >n t^{\prime n-1} f\left(v_{2}\left(t^{\prime}\right)\right)=\left(\left(-v_{2}^{\prime}\left(t^{\prime}\right)\right)^{n}\right)^{\prime} .
\end{aligned}
$$

The continuity of $\left(\left(-v_{1}^{\prime}(t)\right)^{n}\right)^{\prime}$ and $\left(\left(-v_{2}^{\prime}(t)\right)^{n}\right)^{\prime}$ at $t=t^{\prime}$ implies that there exists a $\delta>0$ such that

$$
\begin{aligned}
&\left(\left(-v_{1}^{\prime}(t)\right)^{n}\right)^{\prime}>\left(\left(-v_{2}^{\prime}(t)\right)^{n}\right)^{\prime}, \\
& \forall t \in\left(t^{\prime}-\delta, t^{\prime}+\delta\right) .
\end{aligned}
$$

Integrate (107) from $t^{\prime}$ to $t \in\left(t^{\prime}, t^{\prime}+\delta\right)$ to obtain

$$
\left(-v_{1}^{\prime}(t)\right)^{n}>\left(-v_{2}^{\prime}(t)\right)^{n}, \quad \forall t \in\left(t^{\prime}, t^{\prime}+\delta\right) .
$$

Now, $\left(C_{2}\right)$ implies that

$$
-v_{1}^{\prime}(t)>-v_{2}^{\prime}(t), \quad \forall t \in\left(t^{\prime}, t^{\prime}+\delta\right) .
$$

That is,

$$
\left(v_{1}(t)-v_{2}(t)\right)^{\prime}<0, \quad \forall t \in\left(t^{\prime}, t^{\prime}+\delta\right) .
$$

Then, $v_{1}(t)-v_{2}(t)$ is decreasing on $t \in\left(t^{\prime}, t^{\prime}+\delta\right)$, which contradicts $t^{\prime}=\min _{t \in[0,1]}\left(v_{1}(t)-v_{2}(t)\right)$. Hence, (105) is true.

\section{Conflict of Interests}

The authors declare that there is no conflict of interests regarding the publication of this paper.

\section{Acknowledgments}

The authors thank the referees for their suggestions. This research is supported by Young Award of Shandong Province (ZR2013AQ008) and the Fund of Science and Technology Plan of Shandong Province (2014GGH201010).

\section{References}

[1] N. D. Kutev, "Nontrivial solutions for the equations of MongeAmpère type," Journal of Mathematical Analysis and Applications, vol. 132, no. 2, pp. 424-433, 1988.
[2] S. Hu and H. Wang, "Convex solutions of boundary value problems arising from Monge-Ampère equations," Discrete and Continuous Dynamical Systems, Series A, vol. 16, no. 3, pp. 705720, 2006.

[3] G. Dai, Eigenvalue, Bifurcation, Existence and Nonexistence of Solutions for Monge-Ampère Equations, Analysis of PDEs, 2012.

[4] H. Wang, "Convex solutions of systems of Monge-Ampère equations," http://arxiv.org/abs/1007.3013.

[5] J. Y. Wang, W. J. Gao, and Z. H. Lin, "Boundary value problems for general second order equations and similarity solutions to the Rayieigh problem," The Tohoku Mathematical Journal. Second Series, vol. 47, no. 3, pp. 327-344, 1995.

[6] F. Wang and Y. An, "Triple nontrivial radial convex solutions of systems of Monge-Ampère equations," Applied Mathematics Letters, vol. 25, no. 1, pp. 88-92, 2012.

[7] H. Wang, "Convex solutions of boundary value problems," Journal of Mathematical Analysis and Applications, vol. 318, no. 1, pp. 246-252, 2006.

[8] H. Wang, "Convex solutions of systems of Monge-Ampre equations," Communications in Applied Analysis, vol. 18, pp. 131146, 2014.

[9] J. V. A. Goncalves and C. A. Santos, "Classical solutions of singular Monge-Ampère equations in a ball," Journal of Mathematical Analysis and Applications, vol. 305, no. 1, pp. 240252, 2005.

[10] A. C. Lazer and P. J. McKenna, "On singular boundary value problems for the Monge-Ampère operator," Journal of Mathematical Analysis and Applications, vol. 197, no. 2, pp. 341-362, 1996.

[11] A. Mohammed, "Singular boundary value problems for the Monge-Ampère equation," Nonlinear Analysis, Theory, Methods \& Applications, vol. 70, no. 1, pp. 457-464, 2009.

[12] A. Mohammed, "Existence and estimates of solutions to a singular Dirichlet problem for the Monge-Ampère equation," Journal of Mathematical Analysis and Applications, vol. 340, no. 2, pp. 1226-1234, 2008.

[13] C. Gutierrez, The Monge-Ampère Equation, Birkhäuser, Basel, Switzerland, 2000.

[14] Z. Zhang and K. Wang, "Existence and non-existence of solutions for a class of Monge-Ampère equations," Journal of Differential Equations, vol. 246, no. 7, pp. 2849-2875, 2009.

[15] D. J. Guo and V. Lakshmikantham, Nonlinear Problems in Abstract Cones, Academic Press, New York, NY, USA, 1988.

[16] D. O'Regan, Theory of Singular Boundary Value Problems, World Scientific, Singapore, 1994.

[17] R. P. Agarwal, D. O'Regan, V. Lakshmikantham, and S. Leela, "Existence of positive solutions for singular initial and boundary value problems via the classical upper and lower solution approach," Nonlinear Analysis: Theory, Methods \& Applications, vol. 50, no. 2, pp. 215-222, 2002.

[18] P. Habets and F. Zanolin, "Upper and lower solutions for a generalized Emden-Fowler equation," Journal of Mathematical Analysis and Applications, vol. 181, no. 3, pp. 684-700, 1994.

[19] Z. Wei, "Positive solution of singular Dirichlet boundary value problems for second order differential equation system," Journal of Mathematical Analysis and Applications, vol. 328, no. 2, pp. 1255-1267, 2007. 


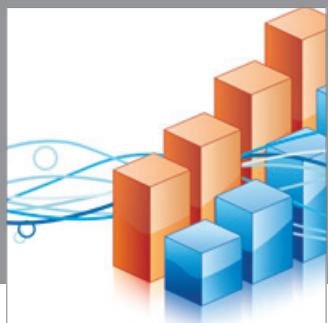

Advances in

Operations Research

mansans

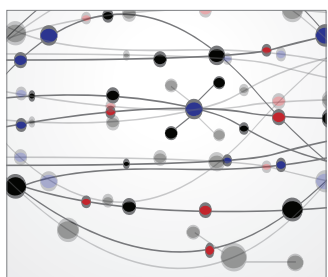

The Scientific World Journal
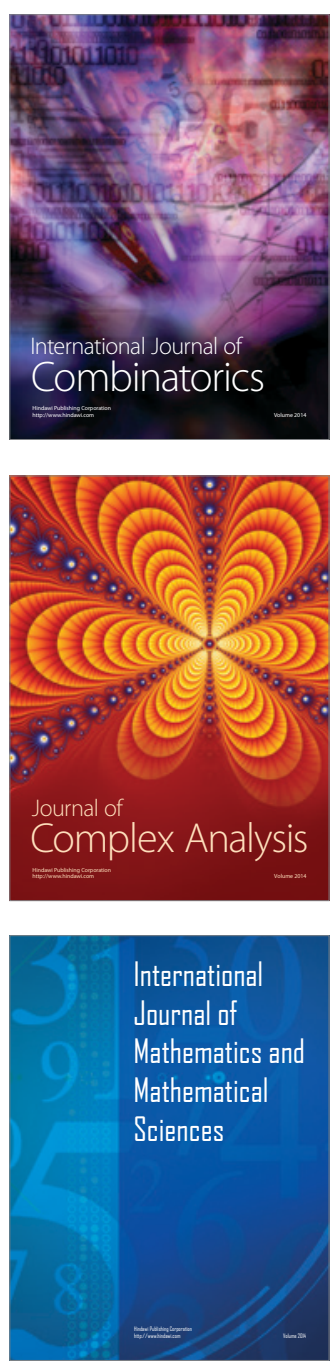
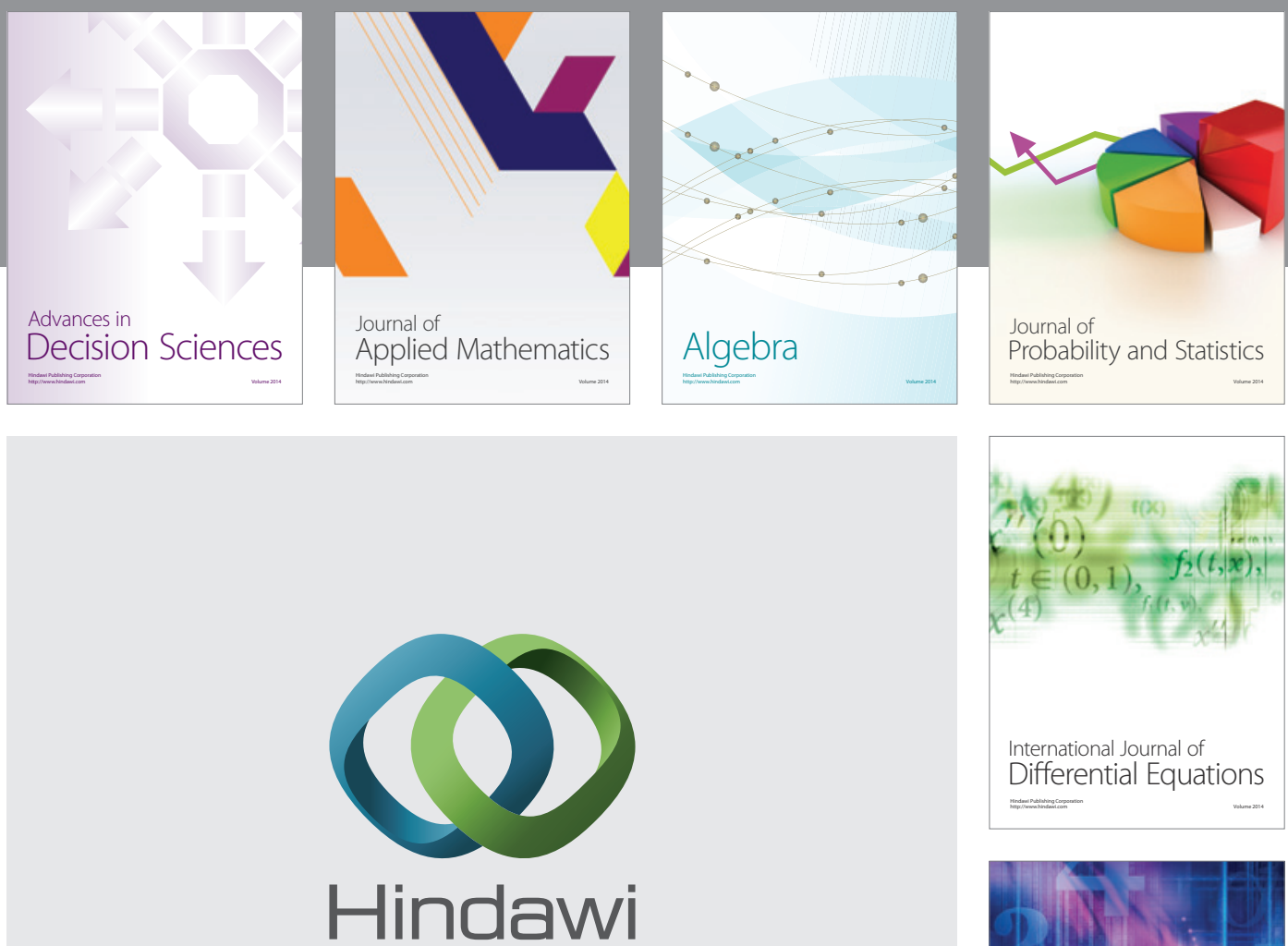

Submit your manuscripts at http://www.hindawi.com
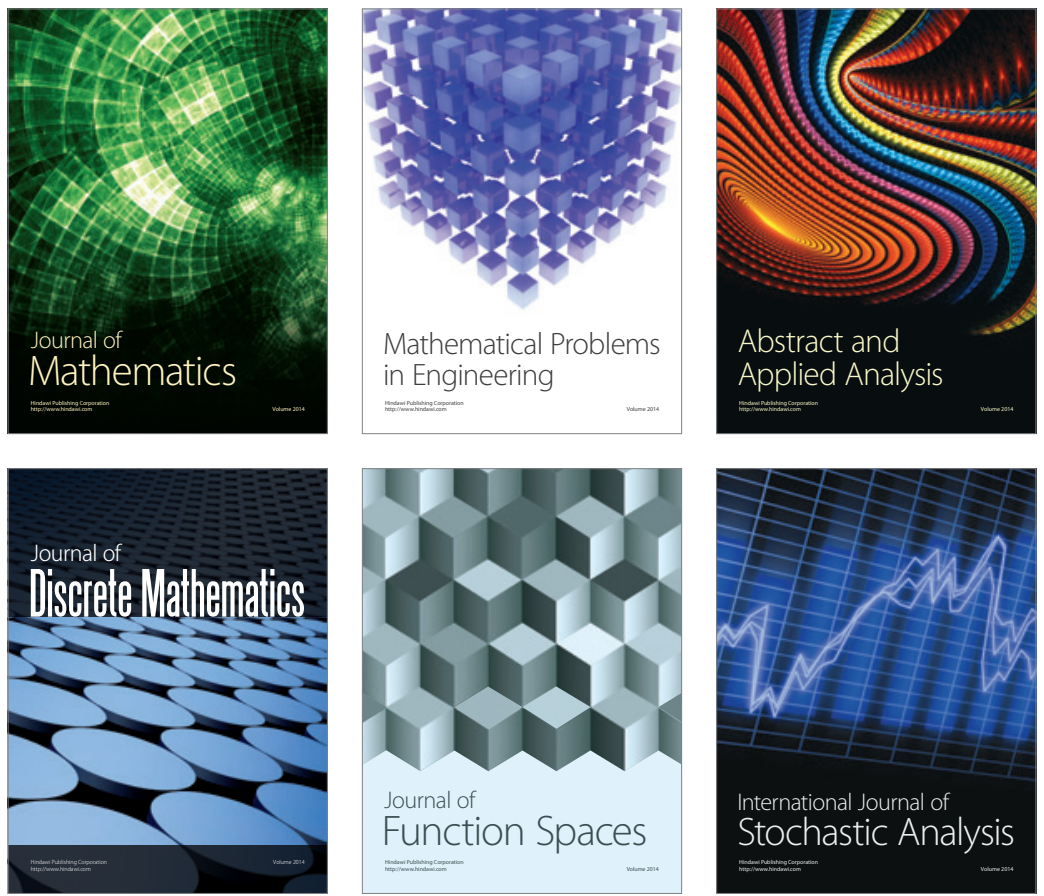

Journal of

Function Spaces

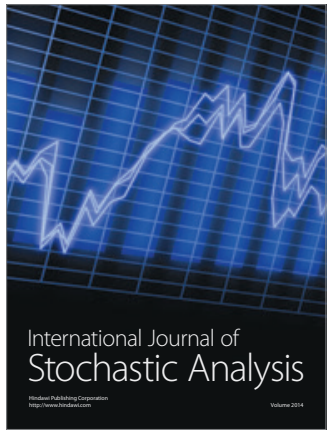

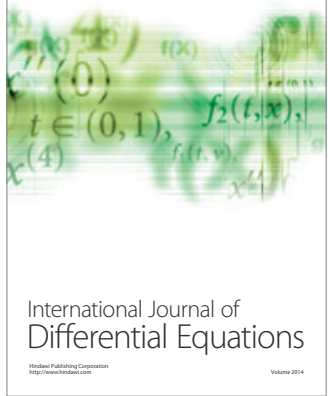
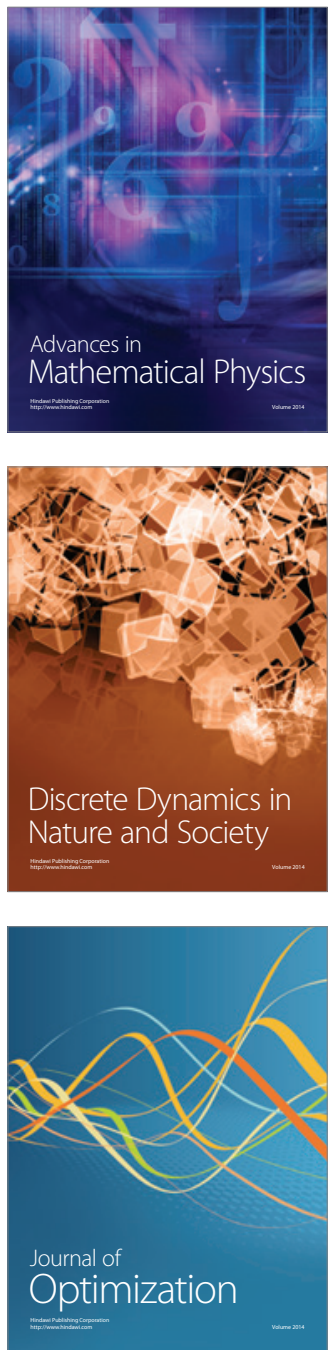